\title{
The NSCL Cyclotron Gas Stopper - Entering Commissioning
}

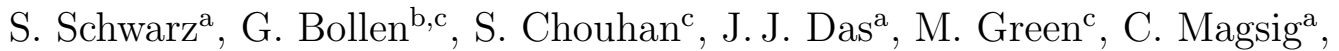
D. J. Morrissey ${ }^{\mathrm{a}, \mathrm{d}}$, J. Ottarson ${ }^{\mathrm{a}}$, C. Sumithrarachchi ${ }^{\mathrm{a}}$, A. C. C. Villari ${ }^{\mathrm{c}}$, A. Zeller ${ }^{\mathrm{C}}$

\footnotetext{
${ }^{a}$ National Superconducting Cyclotron Laboratory, East Lansing, MI, USA

${ }^{b}$ Department of Physics and Astronomy, Michigan State University, East Lansing, MI, USA

${ }^{c}$ Facility for Rare Isotope Beams, East Lansing, MI, USA

${ }^{d}$ Department of Chemistry, Michigan State University, East Lansing, MI 48824, USA
}

\begin{abstract}
Linear gas stopping cells have been used successfully at NSCL to slow down ions produced by projectile fragmentation from the $100 \mathrm{MeV} / \mathrm{u}$ to the $\mathrm{keV}$ energy range. These 'stopped beams' have first been used for low-energy high precision experiments and more recently for NSCLs re-accelerator ReA. A gas-filled reverse cyclotron is currently under construction by the NSCL to complement the existing stopping cells: Due to its extended stopping length, efficient stopping and fast extraction is expected even for light and mediummass ions, which are difficult to thermalize in linear gas cells. The device is based on a $2.6 \mathrm{~T}$ maximum-field cyclotron-type magnet to confine the injected beam while it is slowed down in $\approx 100$ mbar of $\mathrm{LN}_{2}$-temperature helium gas. Once thermalized, the beam will be transported to the center of the device by a traveling-wave RF-carpet system, extracted along the symmetry axis with an ion conveyor and miniature RF-carpets, and accelerated to a few tens of keV of energy for delivery to the users.
\end{abstract}


The superconducting magnet has been constructed on a $60 \mathrm{kV}$ platform and energized to its nominal field strength. The magnet's two cryostats use 3 cryo-refrigerators each and liquid-nitrogen cooled thermal shields to cool the coil pair to superconductivity. This concept, chosen not to have to rely on external liquid helium, has been working well. Measurements of axial and radial field profiles confirm the field calculations. The individual RF-ion guiding components for low-energy ion transport through the device have been tested successfully. The beam stopping chamber with its $0.9 \mathrm{~m}$ diameter RF carpet system and the ion extraction system are being prepared for installation inside the magnet for low-energy ion transport tests.

Keywords:

Gas stopping, Radioactive beams, Cyclotron

\section{Introduction}

At the NSCL rare isotopes are produced by fast projectile fragmentation for a wide range of research. Attractive because of its far reach from the valley of stability and being chemically unselective, the method requires high-energy primary beams to optimize production. Secondary beams are provided by the A1900 fragment separator with energies on the order of 100 $\mathrm{MeV} / \mathrm{u}$. Their large emittance and considerable momentum spread does not allow for efficient electromagnetic deceleration into the keV-energy range. Instead, linear gas stopping cells have been used successfully at NSCL for a decade to slow down these beams; first for use with low-energy high precision experiments such as the Penning-trap mass spectrometer LEBIT and the collinear laser spectroscopy setup BECOLA, and more recently, for NSCL's 
re-accelerator ReA.

For heavy ions, reported stopping efficiencies in linear gas cells have reached $30-40 \%$, depending on beam properties, cell size and operating parameters $[1,2,3]$. However, stopping efficiencies decrease significantly towards lighter masses and with increased beam intensity: Space-charge fields caused by ionization can dominate the electric fields of the ion-guide systems used to transport thermalized ions, thus higher beam currents come with a significant drop in extraction efficiency [4]. For this reason recent generations of gas stopping cells use large volumes $[5,6,7]$ and/or specialized RF ion-guide geometries [8] to mitigate space charge effects.

Due to a combination of low stopping power and large range straggling, stopping lengths of many meters are required to fully contain beams with low atomic number in helium at typical gas pressures of 100 mbar. While constructing linear gas stopping cells with a length of tens of meters may be feasible, this is not attractive for practical reasons and increased extraction times.

\section{NSCL cyclotron gas stopper overview}

The NSCL cyclotron gas stopper takes a different approach to provide sufficient stopping length and volume: Ions are injected at a radius of $\approx$ $0.94 \mathrm{~m}$ into a cyclotron-type dipole magnet, where their momentum is initially degraded to a rigidity of $1.6 \mathrm{Tm}$. The ions start to follow a circular motion: they are confined radially by the dipole field and axially by the varying field strength of the three-sectored poles. In the presence of $\approx 100$ mbar of high-purity helium at $\mathrm{LN}_{2}$-temperature the ions continuously slow 


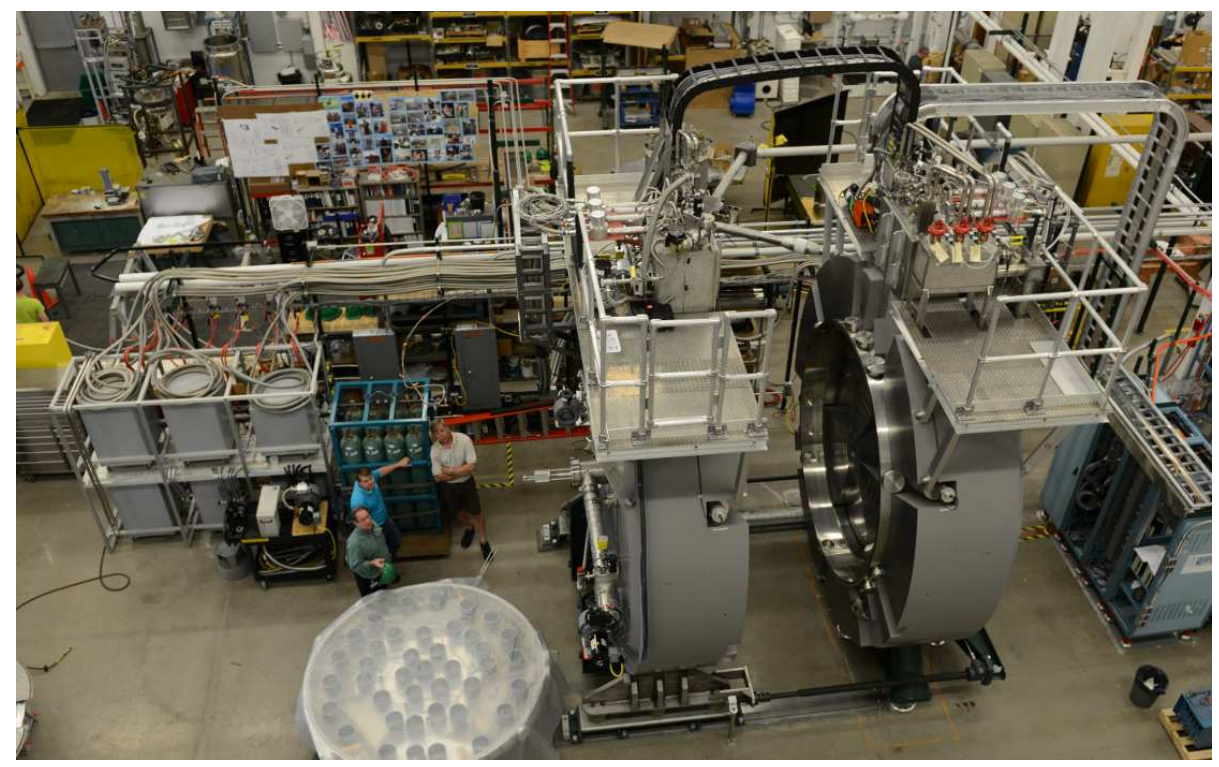

Figure 1: Photo of the cyclotron-stopper magnet with the rolling side retracted for access to the central region. The coils' separate cryostats are at the center of the platforms on top of the magnet halves. The six cryo-compressors powering the cold heads are at the left end of the picture.

down along a spiral path and thermalize in a central region less than $50 \mathrm{~cm}$ in radius. The magnet design is a result of an iterative simulation process with alternating Monte-Carlo-type beam stopping simulations and field calculations. The pole profiles required special attention to avoid beam loss e.g. by resonant coupling of the radial and axial motions $[9,10]$. Simulations have also shown that under conditions that mainly depend on pressure and the incoming ion species, the ions can stop in a central volume that is separated from the region of highest ionization density. In these cases a reduced beam-rate dependence of the extraction efficiency is expected.

The minimum clearance between the poles was increased to $18 \mathrm{~cm}$ in the 
final design to maximize acceptance and allow the installation of the central beam chamber with its $0.9 \mathrm{~m}$-diameter ion carpets. These traveling-wave RF ion carpets [11] will transport the thermalized ions to the center of the device. There the ions will pass through a miniature RF ion carpet, installed for differential pumping purposes, and enter an ion conveyor [12, 13], to transport the ions through the bore of the magnet. The RF ion conveyor is a stack of about a thousand ring-shaped electrodes employing a traveling RF wave with 45-degree phase difference between rings. Despite its complexity, this type of ion guide was chosen as it is expected to transport ions rapidly and efficiently even in the presence of a large magnetic field gradient. Exiting through another RF ion carpet, the ions will be swept into a linear RFquadrupole ion guide for final beam cooling and then accelerated to a few tens of keV of energy for delivery to the users. The cyclotron stopper itself has been placed on a $60 \mathrm{kV}$-tested high voltage stand to provide the accelerating potential.

Combined stopping and extraction simulations indicate that very efficient and fast transmission will be possible even for light and medium-mass ions, see $[14,15]$ for a summary of the calculations. The magnet with its pair of superconducting coils has been constructed on its HV platform and energized to its nominal field strength with a current of $\approx 180 \mathrm{~A}$. Details on the cryostat, the cool-down process and first field measurements are provided in the following two sections. 


\section{Cryogenic system and cool-down}

In order to provide access to the central stopping chamber, the yoke of the magnet was constructed as two halves with separated cryostats: the extraction-side half is fixed to the floor, while the other half can be retracted on a rail system - see Figure 1 for a photo of the system in the open configuration. The two cryostats use three two-stage CryoMech PT415-RM pulse-tube cryo-refrigerators each and liquid-nitrogen cooled thermal shields to cool and maintain the coils in a liquid helium bath. While the cryostats can be connected to a supply of liquid helium to speed up the cool-down process or for a re-fill, the system has enough cooling power to generate and maintain its liquid inventory from a supply of gaseous helium. This concept avoids the problematic transfer of liquid helium to the cryostats when operated on high voltage (HV). Custom HV insulators in the high-pressure He connections to the remote motor units of the cold heads allow the compressors to remain on earth ground.

Figure 2 shows the recorded temperatures during a cool-down of the fixedside cryostat. Liquid-nitrogen cooling of the thermal shields started one day before the three cryo-compressors were started at the time shown as zero. The sensors record temperatures at three different locations of the $2.47-\mathrm{m}$ diameter coil (bottom, side, top), while three more record the second-stage temperatures of the cold heads. This cool-down, performed with a cryostat pressure of 1.6 bar lasted 16 days; other cool-down periods with up to 0.5 bar higher pressure were slightly shorter.

Figure 3 shows the coil temperatures at the end of the cooling period from Figure 2 on an expanded scale and the readout of a helium level probe 


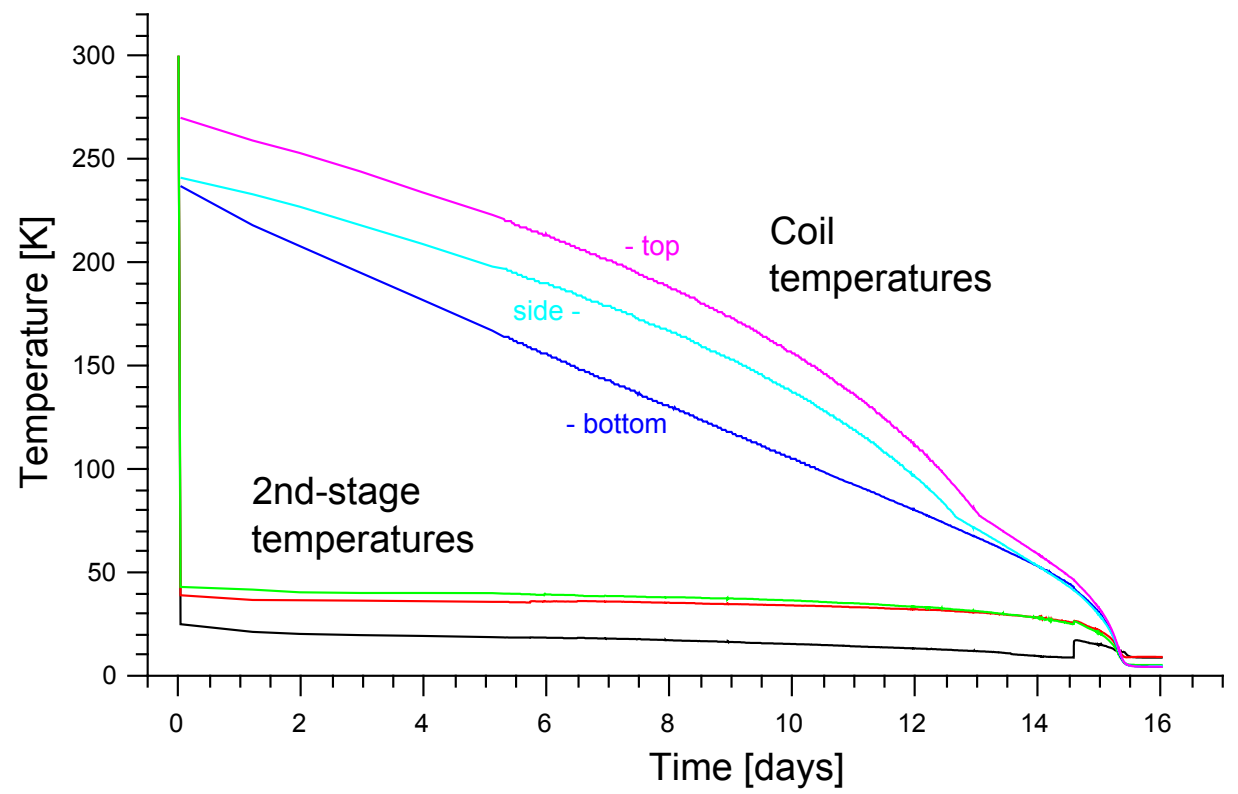

Figure 2: Recorded temperatures during a cool-down of the stationary-side coil from $300 \mathrm{~K}$ to $4 \mathrm{~K}$. The cryo-coolers were turned on at time 0 ; liquid-nitrogen cooling of the shields started a day earlier.

installed in the 'neck' of the cryostat, located above the coil and below the chamber housing the cold heads. Approximately $38 \mathrm{~h}$ after the coil temperatures bottom out at $4.3 \mathrm{~K}$, liquid helium starts to fill up in the neck with a constant rate of $0.28 \mathrm{l} / \mathrm{h}$. The helium space in the neck and around the coil has a design volume of 14.81 , compatible with the fill rate and liquefaction starting at day 16 , at the beginning of the extrapolated line fit to the filling data.

While the cryogenic system has proven to generate the required liquid helium from gas and recondense the helium gas maintaining the liquid inventory, the cool-down time is considerably longer than originally expected. As analyzed in [16], the cool-down time depends, among other parameters, 


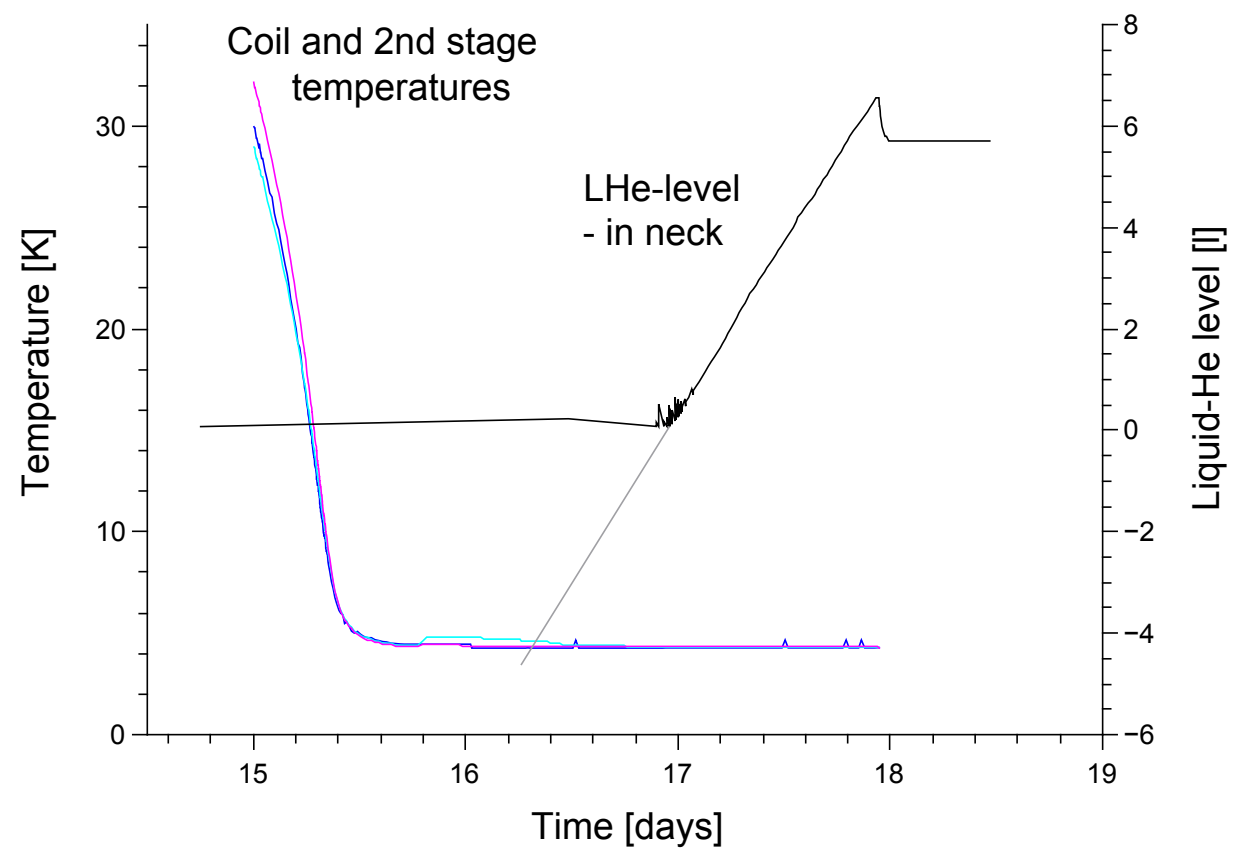

Figure 3: Helium liquefaction at the end of the cooling period shown in figure 2. The installed helium meter measures the level in the neck of the cryostat above the coil. It starts to show liquid $\approx 1.6$ days after the coil temperatures bottom out near $4.5 \mathrm{~K}$. Liquid helium starts to accumulate in the coil at day 16 , at the beginning of the extrapolated fill line. 
critically on the cryostat He-pressure and details in the piping of the thermalsiphon cooling loop. A speed up of the cooling process e.g. by five days would require a cryostat pressure of 6 bars, demanding a redesign of the internal helium piping.

During initial commissioning tests in 2014 the heat load on both cryostats was very close to the capacity of the six cold heads (rated at least $1.35 \mathrm{~W}$ at their second stages with no load on their first stages). The pipes feeding room-temperature gas to the He-cryostats at the second stages of the cold heads were identified as a source of excess heat, re-routed and better thermally intercepted at the cryostats' $80 \mathrm{~K}$ and $40 \mathrm{~K}$ shields. Following adjustments to the coils' support links, made to balance forces as the magnet current was gradually increased to its nominal value (see below), the heat load was reduced considerably and both cryostats can now maintain liquid helium with only two of their three cryo-refrigerators running. With all cold heads operating, PID pressure controllers add between 0.7 to $1.7 \mathrm{~W}$ of heat to each of the cryogenic systems to maintain a pressure of 0.14 bar above atmospheric. More details on the cryogenic system of the cyclotron stopper can be found in $[16,17,18]$.

\section{Magnet commissioning and field measurements}

With the cryogenic system functional and the two coils superconducting, magnet tests started in fall 2014. A series of tests at lower currents were conducted to ensure that the magnet's protection system was functional. As detailed in [10], the system uses a two-tiered shutdown scheme to protect the magnet: Indications of unbalanced loads in the two coils, excessive tempera- 
tures, lead or support link failures will cause the protection system to induce a safe quench and remove the stored energy within a few minutes. This is called a 'fast dump'; meant to protect the coils from structural damage. Indications of less serious failures, i.e. declining helium-levels, can trigger a 'slow dump', in which the coil currents are discharged through a series of high-power diodes instead of the magnet's four-quadrant power supply. Both protection schemes have been tested successfully. The fast dump essentially resets the fill level and temperatures of the cryostats to a point roughly equivalent to day 15.5 in Figures 2 and 3; i.e. following these events it took about two days before the liquid helium was restored and the magnet was ready for operation again.

The coils' axial and radial support links were adjusted in a sequence of ramping exercises with increasing peak current until the nominal operating current of $180 \mathrm{~A}$ was reached with balanced forces. Figure 4 shows calculated and measured axial magnetic field components at $180 \mathrm{~A}$ current. The measurements were taken with a Metrolab THM1176 3-axis Hall probe, mounted on a slide that allowed approximately $1.2 \mathrm{~m}$ of linear travel. The top figure shows radial scans across a hill and a valley; the bottom figure shows an axial scan along the central bore of the magnet. The two inset figures illustrate the paths followed for the field data. In the radial scans the field strength peaks near $2.6 \mathrm{~T}$, outside the central $0.2 \mathrm{~T}$ depression, which is due to the axial opening in the return yoke. The field along the hill then drops to about $2 \mathrm{~T}$, while the field on the valley drops to $\approx 1.4 \mathrm{~T}$. Both profiles then converge towards zero magnetic field at the edge of the return yoke. The axial field profile drops from its midplane-maximum at $2.36 \mathrm{~T}$ to $\approx 12 \mathrm{mT}$ at the edge 

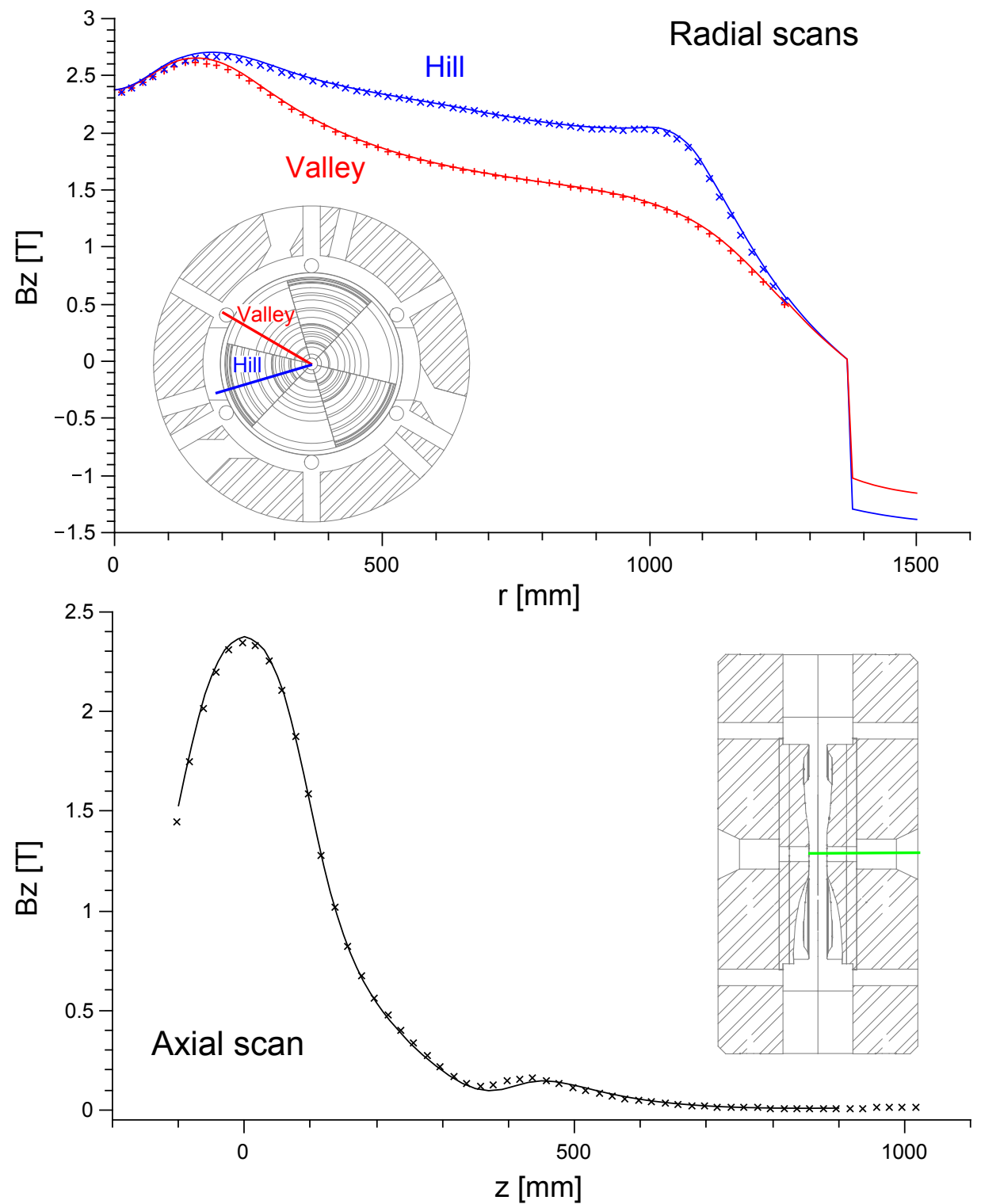

Figure 4: Calculated (lines) and measured (dots) axial field components at the nominal magnet operating current of $180 \mathrm{~A}$. The top figure shows radial scans following paths along a hill and a valley; the bottom figure shows an axial scan through the bore of the magnet. The solid lines in the inserted sketches indicate the paths followed for the field data. 
of the magnet. The axial profile exhibits a bump at $z=430 \mathrm{~mm}$, caused by a change in bore diameter from $150 \mathrm{~mm}$ to $300 \mathrm{~mm}$ at $z=388 \mathrm{~mm}$.

Within the accuracy of the Hall probe, specified to better than $\pm 1 \%$, the radial-scan data agree very well with the TOSCA 3D field calculations [10] within the pole segments. Consequently, the magnetic field index derived from the measured data agrees well with expectations. Beyond a radius of $0.25 \mathrm{~m}$ the field index stays clear of critical values that could lead to undesired resonant excitations of motion during the slowing-down process. The axialscan data agree within $2 \%$ for field strengths above $0.5 \mathrm{~T}$; below that, the agreement is within $30 \mathrm{mT}$. Ion transport through the conveyor inside the bore is expected to be insensitive to details of the magnetic field profile [13].

\section{Commissioning status; ion extraction}

The cyclotron stopper is currently installed in an assembly area, separated from NSCL's beam lines, in order to allow low-energy beam operation during commissioning activities. Tests of the cyclotron stopper in its current off-line location will conclude when ion transport along the central RF ion carpets and through the extraction system has been demonstrated at the full magnetic field. With the magnet successfully operated at its design field, efforts are now shifting back to ion transport tests.

The concept of ion transport along the large-scale traveling-wave carpets has been tested extensively with prototypes of similar size before, see [11, 13] for details. To cover the expected volume of thermalized ions, the cyclotron stopper will use a $0.9 \mathrm{~m}$ outer-diameter RF ion carpet in its central chamber - see Figure 5 for a photograph. Because of its large capacitive load 


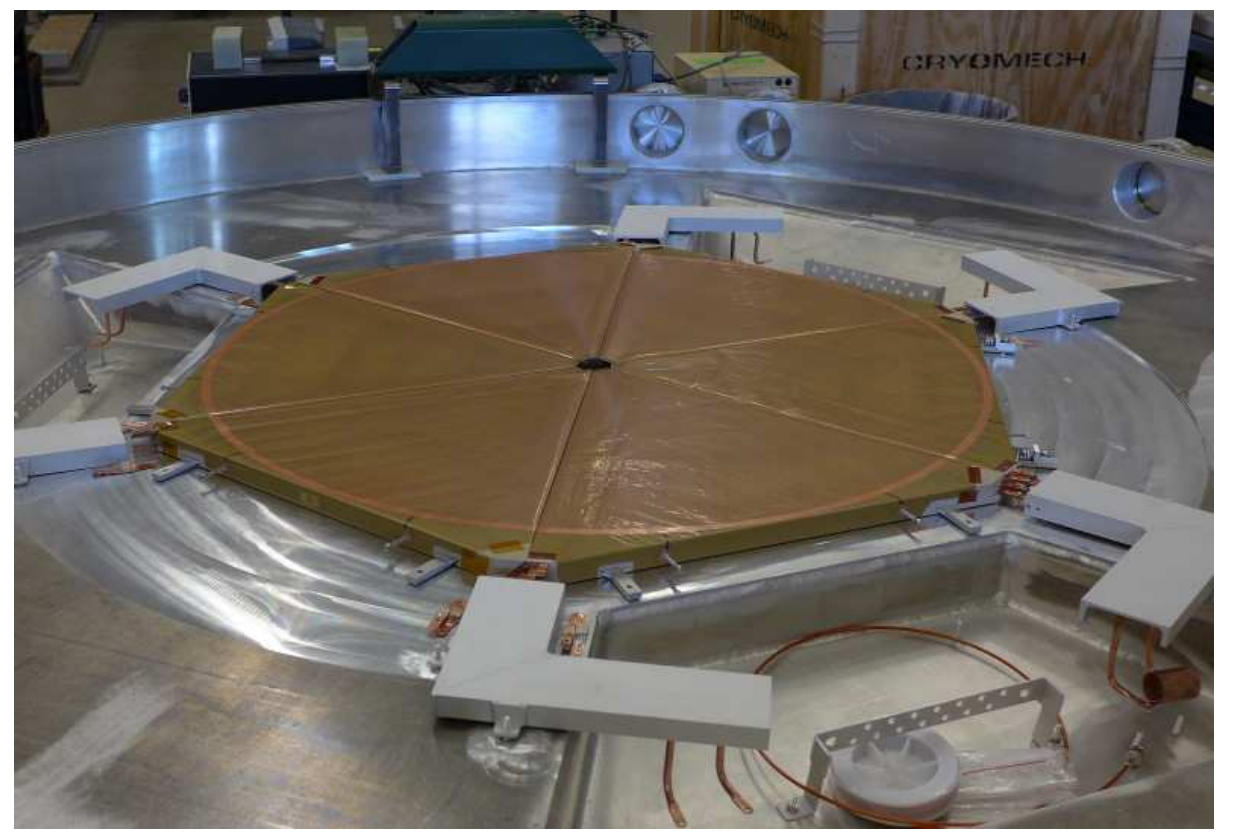

Figure 5: Photograph of the sixfold-segmented main RF carpet in the central chamber of the cyclotron gas stopper. 
and for practical reasons, the carpet has been built in six $60^{\circ}$ sectors with separate resonant RF driver circuits, capable of delivering the required RF amplitudes at $7 \mathrm{MHz}$ frequency. To keep RF losses to a minimum, the resonant circuits have been placed close to the carpets, in pockets added to the vacuum chamber, which make use of the space in the pole valleys.

The liquid-nitrogen cooled central vacuum chamber, which houses the large-scale carpets, is being prepared for installation. Due to its large $2.35 \mathrm{~m}$ diameter, the lids of the chamber will not tolerate significant pressure differences between the inside and the surrounding guard vacuum. A pressure bypass system has been designed and installed to protect the chamber during pumping cycles and during operation at $100 \mathrm{mbar}$ RT-equivalent pressure.

As reported in [12], the ion conveyor system with its entrance and exit mini-carpets are being tested in NSCL's stopping vault to benefit from existing pumping and diagnostics infrastructure. The expected pressure drops across the mini-carpet pumping barriers have been confirmed and initial ion transport tests through the $1 \mathrm{~m}$ long ion conveyor have demonstrated efficiencies exceeding $80 \%$.

Following the installation of all carpets and the ion conveyor in the cyclotron stopper, ion transport tests along the entire extraction system will start. A movable test ion source has been designed and will be installed on an arm reaching across the main RF carpets. This setup will allow systematic transport tests along the main RF carpets in the presence of magnetic field and helium gas. A final test with the source placed at the entrance of the conveyor and reduced gas pressure will test the ion conveyor's performance in the presence of the magnetic field gradient. 


\section{Acknowledgements}

The authors acknowledge support from the National Science Foundation under grants PHY-09-58726 and PHY-11-02511 as well as support from Michigan State University.

\section{References}

[1] W. Plaß et al, Nucl. Instr. Methods B 317 (2013) 457-462.

[2] M. Reiter et al, in: these proceedings.

[3] C. Sumithrarachchi et al, in: these proceedings.

[4] D. Morrissey, European Physical Journal, Special Topics 150 (2007) 365.

[5] M. Petrick et al, Nucl. Instr. Methods B 266 (2008) 4493.

[6] M. Wada et al, Nucl. Instr. Methods B 204 (2003) 570581.

[7] G. Savard, Journal of Physics: Conference Series 312 (2010) 052004.

[8] K. Cooper et al, Nucl. Instr. Methods A, 763 (2014) 543.

[9] S. Chouhan et al, in: Proceedings 22nd Particle Accelerator Conference, PAC07, Albuquerque, NM, USA, 6/25-29/2007, p. MOPAS041.

[10] S. Chouhan et al, IEEE Transactions On Applied Superconductivity 23 (2013) 4101805.

[11] A. Gehring et al, in: these proceedings.

[12] A. Villari et al, Nucl. Instr. Methods B, these proceedings. 
[13] M. Brodeur et al, Nucl. Instr. Methods B 317 (2013) 468-472.

[14] S. Schwarz et al, Nucl. Instr. Methods B 317 (2013) 463-467.

[15] N. Joshi et al., in: Proceedings of IPAC2012, New Orleans, USA, jacow.org

[16] M. Green et al, in: Proc. Cryogenic Engineering Conference and International Cryogenic Materials Conference CEC/ICMC 2015, Tucson, AZ, 6/28 - 7/2, 2015, IOP Conference Series, submitted.

[17] S. Chouhan et al, in: Proc. North-American Particle Accelerator Conf., Pasadena, CA, NA-PAC'13, 9/29 - 10/4, 2013, jacow.org, p. THPBA12.

[18] M. Green et al, in: Proc. Sixth International Particle Accelerator Conference, Richmond, VA, 5/3 - 5/8, 2015, jacow.org, accepted. 